\title{
Sliver Solar Cell Technology: Pushing the Material Boundaries
}

Evan Franklin, Andrew Blakers, Klaus Weber, Vernie Everett The Australian National University, Canberra, ACT 0200 Australia.

\begin{abstract}
One of the primary objectives of the global photovoltaic research community is to effect significant manufacturing cost reductions, either by reducing material and processing costs or by increasing solar cell efficiency. One very promising technology for achieving both of these goals is Sliver technology, which offers potential for a 10- to 20-fold reduction in the consumption of purified silicon, while at the same time achieving very high cell efficiencies by fully exploiting the advantages of mono-crystalline silicon.

Sliver solar cells are thin, mono-crystalline silicon solar cells fabricated using a combination of micro-machining techniques and standard silicon device fabrication technologies. Rather than fabricating a single solar cell on the surface of a wafer, many hundreds to several thousand individual Sliver solar cells are fabricated within a single wafer. The dimensions of a Sliver cell depend upon wafer size, wafer thickness, and the micro-machining method employed. Cells typically have a length of $5-12 \mathrm{~cm}$, a width of $0.5-2 \mathrm{~mm}$, and a thickness of $20-60$ micron. 20\% efficient Sliver solar cells using standard cell processing methods and a robust processing sequence, have been fabricated at ANU. Current research efforts are directed towards developing and establishing new fabrication techniques to further simplify the fabrication sequence and to improve cell efficiency.

This paper presents an overview of Sliver technology. The fabrication method and some key challenges in producing Sliver cells is presented along with the measured performance of cells fabricated in the ANU solar research laboratory.
\end{abstract}

\section{INTRODUCTION}

The global photovoltaic market has grown at well over $40 \%$ per annum over the past decade, with estimated production of $27.2 \mathrm{GW}$ [1] and a reported installed capacity of $18.2 \mathrm{GW}$ [2] in 2010. During this period of growth, the manufacturing base has expanded from a relatively small number of players, typically linked to existing large corporations with a wide variety of interests (BP, Shell and Sharp for example), to a number of large and specialized photovoltaic manufacturers with interest across many parts of the entire solar cell value chain, from silicon feedstock to solar system sales (REC, Trina and Suntech for example). The rapid growth within the industry, coupled with continual advances in solar cell technology, has gone hand-in-hand with a decline in costs, as demonstrated by the often cited photovoltaic 'learning curve', a recent update of which shows a long-term trend learning rate of around 20\% [3]. It remains to be seen, as the industry continues to grow and mature, how far and how fast costs will keep declining. One thing however is clear: there is the ongoing need to find cost reductions through manufacturing scale and efficiency, and through advances in technology.

The standard solar cell technologies produced in industry, with over $85 \%$ share, are ones based on wafered crystalline silicon [1]. Wafer-based technologies are all essentially concerned with processing the surfaces of the wafer, with the processed regions occupying an area within a few microns only of the wafer surface. The main pathway to increased silicon utilization is to produce thinner wafers with minimum kerf loss, provided that they are still able to be processed into solar cells with high yield; currently the industry appears to have settled on a wafer 
thickness of about $180 \mu \mathrm{m}$, with wafering kerf loss also approaching this value. The commonly employed manufacturing process uses an homogenous front-surface phosphorus emitter, PECVD silicon nitride ARC, screen printed front fingers and a full rear screen-printed aluminium back surface field. Manufacturers produce approximately $16 \%$ and $17 \%$ efficient solar cells on multicrystalline mono-crystalline wafers respectively. Further incremental efficiency gains can be made by implementing one of several proposed selective emitter schemes [4-7], localized rear contact schemes [8], or by one of the various technologies which moves the front contacts to the rear of the cell $[9,10]$. The highest recorded silicon solar cell efficiency in the lab is $25.1 \%$ [11].

Thin film photovoltaic technologies, of which there are many variants, are recently gaining traction in industry, most notably CdTe technology with approximately $5 \%$ of total market share through the company First Solar [1], although market penetration has been largely restricted to utility scale solar farms. Thin film technologies feature much lower material costs, when compared to wafer based technology, with typically only a few microns of material deposited to create the active cell area. Solar conversion efficiencies of thin-film technologies are generally considerably lower than for wafer-based technology. Some questions have been raised about the long-term viability of raw materials supply for some of these technologies [12]. Despite the undeniable promise of thin-film technologies, it is not clear yet whether they will be capable of producing energy at lowest cost for the bulk of the market in the longer term. A comparison of the general characteristics of wafer-based and thin-film technologies, and also Sliver technology, is given in table I.

Table I. A comparison of characteristics of silicon wafer-based and thin-film solar technologies, with the potential of Sliver technology.

\begin{tabular}{lll}
\hline Wafer-based silion & Thin-film technology & Sliver technology \\
\hline Low Capex & High Capex & Low Capex \\
Abundant metrials supply & Some materials limitations & Abundant materials supply \\
Material usage high & Material usage low & Material usage low \\
Higher efficiency & Moderate efficiency & Higher efficiency \\
Accepted in all markets & Acceptance in some markets & Known product type \\
Standard product type & Broader suite of product & Broader suite of product \\
\hline
\end{tabular}

Sliver technology aims to take the best characteristics of wafer-based silicon technology and combine them with many of the advantages of thin-film technology. The technology uses crystalline silicon, conventional processing techniques and equipment, is inherently designed to yield high-efficiencies, results in a finished module that is similar to traditional silicon based products, but utilises material in a similar manner to thin-film technologies and has the potential for a wider range of products such as flexible modules and a variety of shapes and sizes. Insofar as the technology is capable of realising substantial reductions in silicon consumption, Sliver technology can be compared to those thin-film technologies based on silicon, such as those developed at Fraunhofer ISE [13], ISFH [14] and UNSW [15]. Sliver technology makes better utilization of silicon in the same way as these technologies do, but has the major advantage of using standard wafer-based silicon processing technologies while also yielding high efficiency solar cells. Sliver cells are still fabricated from crystalline silicon wafers but in a radically different way to other technologies. Rather than using only the surfaces of the wafer to form the solar cell, sliver cells are fabricated using the whole volume of a wafer. The Sliver technology was developed [16-19] at the Centre for Sustainable Energy Systems at the Australian National 
University (ANU), supported by the Australian company Origin Energy and is now the subject of a major commercialization effort by the Idaho, USA, based company Transform Solar [20].

Figure 1 represents a wafer in which sliver cells have been created. The essential step in forming slivers is the micromachining of deep, narrow grooves all the way through a thick silicon wafer. The wafer is then processed using standard techniques to turn each of the slivers into a solar cell. Completed cells are removed from the wafer and rotated through 90 degrees to give a large area multiplication. The large face of the sliver cell, formed by grooving, becomes the sun-facing surface of the cell, whilst the metalized contacts run along either edge. This surface is referred to as the Sliver 'sidewall' throughout this paper. Since the processing treatment of both sidewalls of a sliver is identical, Sliver cells are perfectly bifacial.

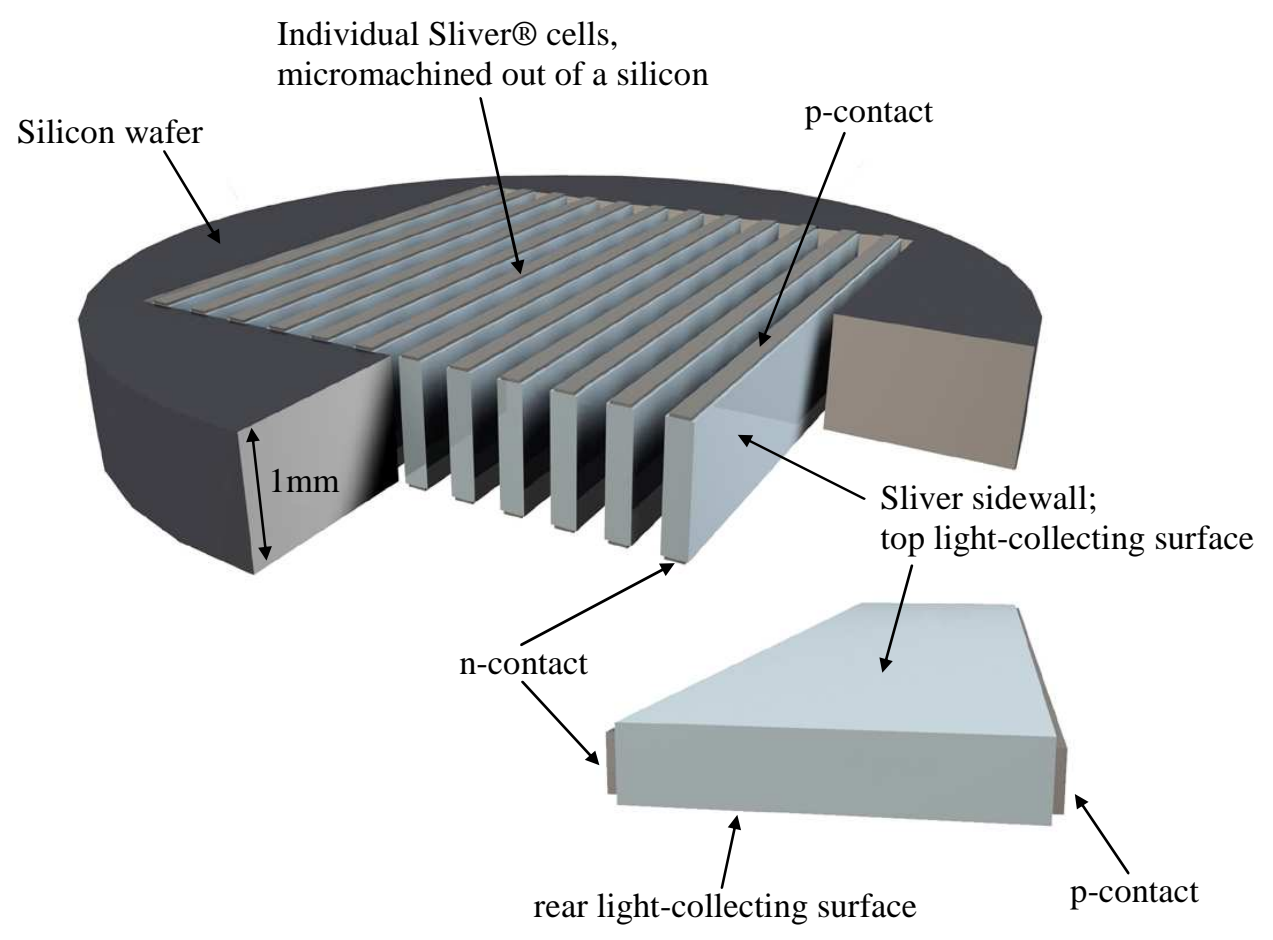

Figure 1. A wafer containing Sliver cells.

The total active solar cell area that can be created from a single wafer is no longer determined by the surface area of that wafer. Instead, many hundreds or even thousands of individual solar cells can be fabricated within a single wafer to yield a total active surface area many times larger than the original wafer area and with much higher utilization of the silicon volume. The dimensions of a Sliver cell depend upon wafer size, wafer thickness and sliver patterning method; cells typically have a length of $5-10 \mathrm{~cm}$, a width of $1-2 \mathrm{~mm}$, and a thickness of $20-60 \mu \mathrm{m}$. The cells are symmetrical, bi-facial, and quite flexible and strong. The technology allows for a 10- to 20-fold decrease in silicon utilization, and a 20- to 40-fold reduction in the numbers of wafers processed per MW, compared to standard wafer technology.

In addition to the high material utilization at the solar cell fabrication stage, further significant reductions in material and manufacturing costs can be achieved by clever module designs, made possible by the unique size, shape, and operating characteristics of Sliver cells. A module with rear lambertian reflector and evenly spaced-out Sliver cells can, for example, capture over $80 \%$ of incident light with only $50 \%$ of the area being covered by cells [21], again increasing the total solar module area that can be produced per unit of silicon. 


\section{EXPERIMENT}

The requirements for the fabrication of sliver cells are more involved than for conventional high efficiency solar cell cells: a larger number of processing steps is required, and some particular steps necessitate adaptations of existing techniques. Naturally, in designing a suitable sliver cell fabrication process there is an incentive to reduce complexity to produce a short sequence. Complex wafer processing is generally more expensive since it entails a larger fabrication facility, more processing equipment, and higher operation and maintenance costs, while a longer processing sequence will generally result in a lower yield compared to a similar but shorter sequence. Fortunately however, a comparatively long and complex wafer process with good process control can be afforded for Sliver technology since each wafer processed can yield a far greater solar cell output than for conventional technology. A single $15 \mathrm{~cm}$ diameter host wafer can, for example, contain enough Sliver cells to populate a module with a rating of up to $100 \mathrm{~W}$; in comparison, the same ingot which has been trimmed to produce $12.5 \mathrm{x} 12.5 \mathrm{~cm}$ pseudo-squared wafers currently yields in the order of $2.5 \mathrm{~W}$ per wafer processed.

\section{Sliver cell processing overview}

There are a variety of possible fabrication sequences that may be employed to produce sliver cells. The fundamental processing requirements, in no particular order, are: the wafer must be grooved to form individual slivers, with smallest pitch possible; the grooved sidewall regions must be diffused to form $\mathrm{p}-\mathrm{n}$ junctions across each entire surface; the two wafer surfaces (and hence Sliver edges) must be heavily doped, with opposite polarities, to facilitate good ohmic contacts with minimal recombination; and the two wafer surfaces (and hence Sliver edges) must be metallised at least partially. These requirements are all realisable using standard photovoltaic or semiconductor processing equipment and techniques, albeit tailored to the Sliver technology. The processing step which sets Sliver technology apart from other solar cell technologies is the wafer grooving step. Meanwhile, the presence of these deep grooves in turn impacts upon how many of the other processing steps are managed. Of particular concern are three key fabrication processes unique to the Sliver technology or requiring special tailoring and management: groove formation, surface texturing, and silicon doping. These three processes are dealt with in some detail in this paper.

The processing sequence most successfully employed at ANU to date is summarised in Table II. This sequence has several advantages over other fabrication methods: groove formation takes place late in the process thus reducing handling of slivered wafers, doping is done by via diffusions requiring no special lithographically defined localisation, and both negative and positive contacts are defined at the same time also without the requirement for alignment. Doping is typically conducted in $\mathrm{POCl}_{3}$ and $\mathrm{BBr}_{3}$ tube diffusion furnaces, with the sidewall diffusion requiring particular techniques unique to the sliver wafer topology. Wafer cleaning and etching, and the selective one-sided removal of diffusions, is done via wet chemistry long established in solar cell manufacturing. Texturing is done via a process developed at ANU specifically for the Sliver technology, since conventional techniques cannot be applied readily with the sliver topology. Anti-reflection coating and surface passivation is achieved by a dielectric stack consisting of a thin thermal silicon dioxide below an LPCVD silicon nitride film. Opening up of contact areas in the dielectric on both top and bottom of the wafer is done via directional reactive ion etching, which may be masked to open up any desired metal contact fraction. Metallisation itself is conducted by metal evaporation and can be thickened if needed by metal plating. 
Table II. A preferred Sliver cell processing sequence overview [22].

\begin{tabular}{cl}
\hline Step number & Step description \\
\hline 1 & Wafer etch and clean \\
2 & Heavy phosphorus diffusion and in-situ oxide growth \\
3 & Selective removal of diffusion from one wafer surface \\
4 & Heavy boron diffusion \\
5 & Groove mask formation \\
6 & Initiate grooves \\
7 & Groove formation \\
8 & Sidewall texture \\
9 & Sidewall emitter phosphorus diffusion \\
10 & ARC \& surface passivation growth / deposition \\
11 & Remove dielectric from both wafer surfaces \\
12 & Metalize wafer surfaces (which become the Sliver cell edges)
\end{tabular}

\section{Groove formation}

The ideal groove formation process is one that creates, at low cost, the largest number of slivers for a given wafer size and is suited to a high throughput manufacturing environment, while preserving silicon's electronic properties. Many methods can be employed and several of these have been used at ANU for the formation of grooved Sliver wafers. Possible methods include dicing saw, dry or waterjet guided laser systems, wet chemical anisotropic etching, and directional plasma etching. However, many of these methods have been discovered to be slow, be limited in terms of achievable groove depth, or can be demonstrated to result in considerable silicon damage Investigations to date have led to anisotropic wet etching being considered the most suitable method. It is also a low cost process, requiring relatively small volumes of low temperature etch solution to process many wafers simultaneously. The main disadvantage of the anisotropic etching method, over some other methods which are independent of crystallography, is the requirement for a non-standard surface texturing process after groove formation.

The basis for anisotropic wet chemical etch formation of sliver wafers is the exploitation of the differential etch rate of the major families of primary silicon crystal planes in hydroxide etch solutions. The three most commonly used anisotropic etch solutions are sodium hydroxide $(\mathrm{NaOH})$, potassium hydroxide $(\mathrm{KOH})$ and tetramethyl ammonium hydroxide $\left(\mathrm{CH}_{3}\right)_{4} \mathrm{NOH}$, commonly referred to as TMAH). The etch rates of $\{100\}$ and $\{110\}$ planes are much higher in these solutions than $\{111\}$ planes, depending upon concentration and temperature of the solution, and also on the dopant or impurity levels in the silicon itself. Figure 2 shows the major crystal planes relevant for deep groove formation in silicon wafers. Owing to the angles between planes, a wafer oriented with a $\{110\}$ wafer surface will have two $\{111\}$ planes that are exactly perpendicular to the wafer surfaces. Thus, a suitable etch mask which restricts etching to narrow openings each parallel to one of these planes can result in deep narrow grooves forming. Etching will be effectively terminated along the length of the groove by the exposed $\{111\}$ crystal plane and at each end of the groove by two other exposed $\{111\}$ planes, and if allowed to continue will result in grooves being machined completely through the wafer. The strip of silicon remaining after groove formation, which later becomes an individual sliver cell, therefore has sidewalls of $\{111\}$ orientation. Figure 3 is a photo of a completely etched sliver wafer. The semi-transparent 
central square area is where the etching has taken place contains many hundreds of etched strips of silicon.

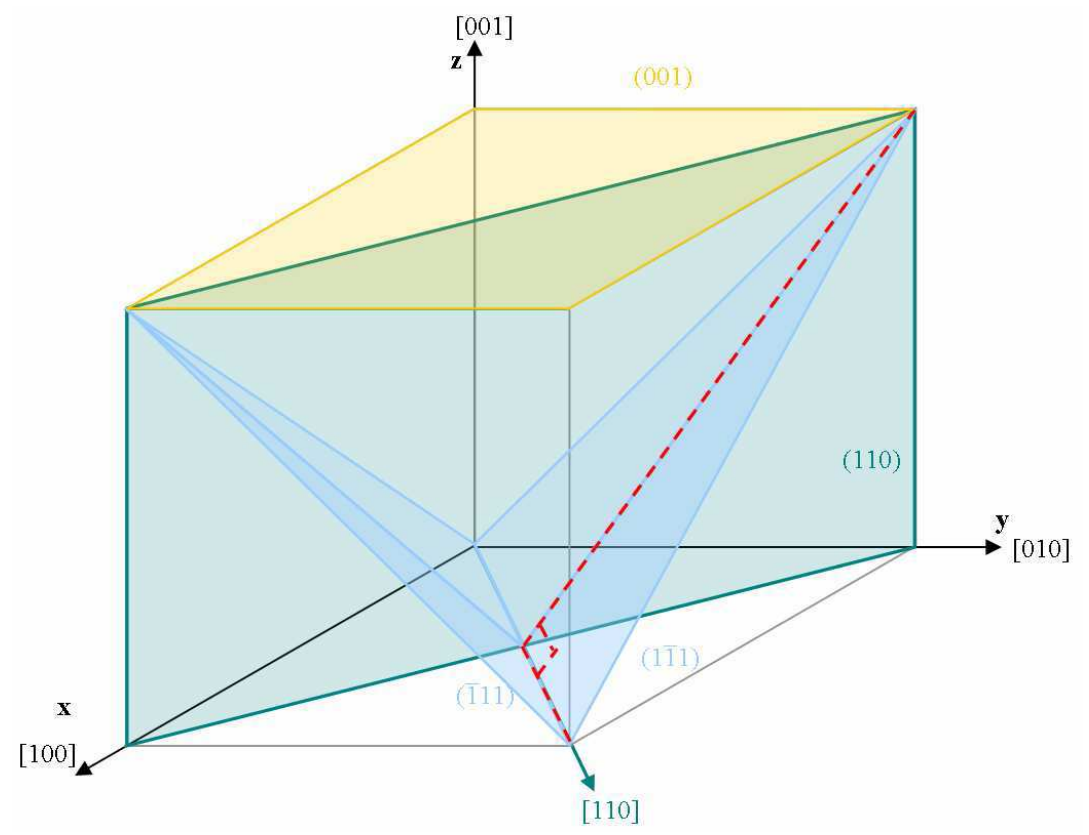

Figure 2. The silicon crystal planes relevant to anisotropic Sliver groove etching. Each $\{110\}$ plane has two $\{111\}$ crystal planes that intersect perpendicular to it.

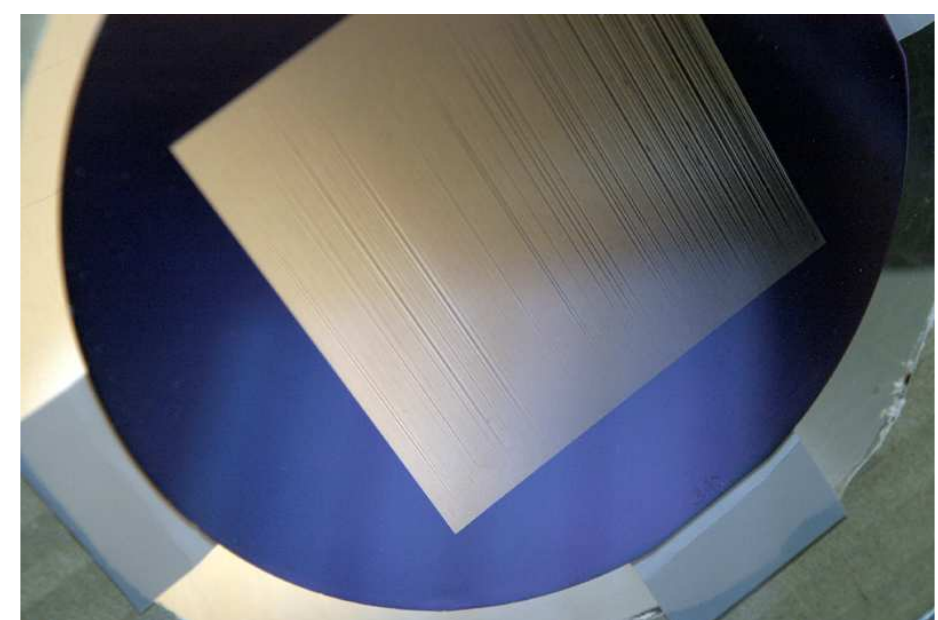

Figure 3. A sliver wafer containing many hundreds of chemically etched strips of silicon, which are subsequently processed to become Sliver cells.

The higher the anisotropy of an etch solution - the ratio of $\{110\}$ to $\{111\}$ crystal plane etch rates - the more closely spaced together the grooves can be formed, and hence the more sliver cells can be produced and the larger the effective wafer area multiplication. Wafer area multiplication (the ratio of sliver cell area produced from a single wafer compared to that wafer area) is plotted in Figure 4 as a function of anisotropy and for several different wafer and sliver thicknesses. In this case it is assumed that only $80 \%$ of the wafer surface itself is patterned to form Sliver cells; this is approaching the practical limit for the technology. 


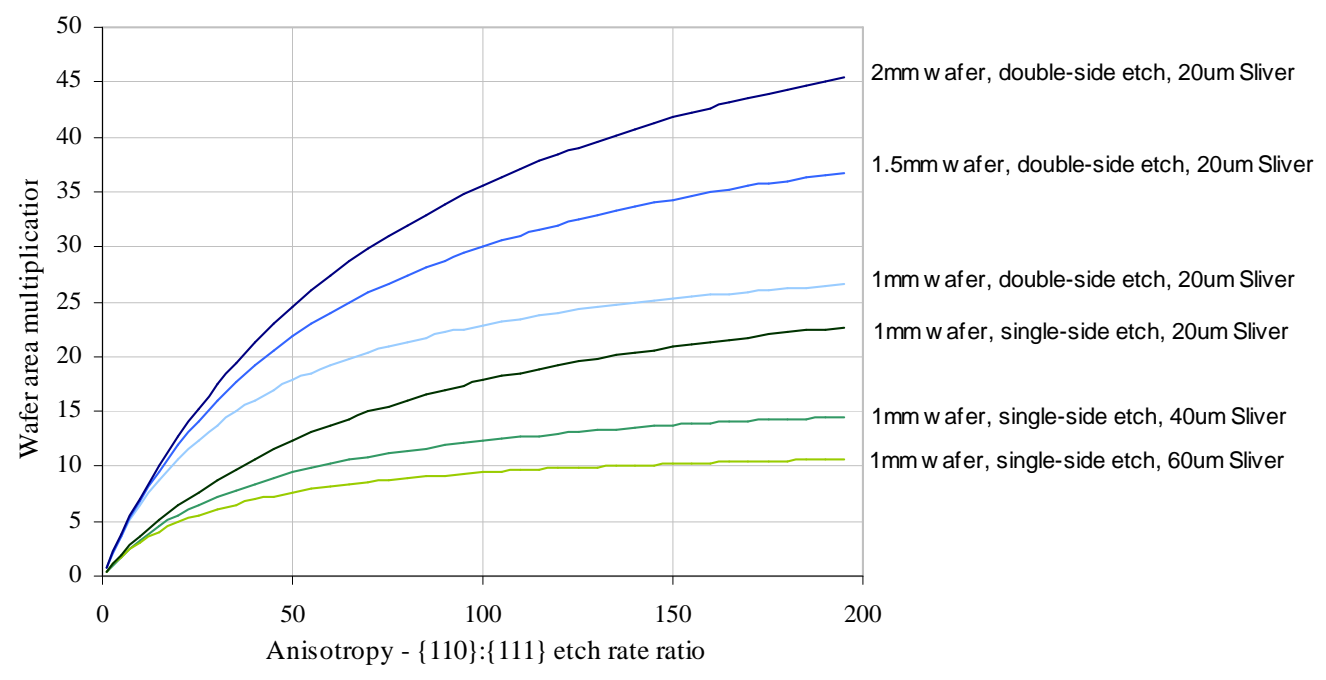

Figure 4. Wafer area multiplication as a function of etch anisotropy. Calculations based on initial groove opening of 5 um and wafer surface pattern fraction of $80 \%$.

Shikida et al. accurately measured etch rates of the various crystal planes in both $\mathrm{KOH}$ and TMAH solutions [23]. They reported a $\{110\}:\{111\}$ anisotropy of 150 for a $36 \% \mathrm{wt} / \mathrm{wt}$ $\mathrm{KOH}$ etch solution at $70^{\circ} \mathrm{C}$, and an anisotropy of 60 for a $25 \% \mathrm{wt} / \mathrm{wt} \mathrm{TMAH}$ etch solution at $85^{\circ} \mathrm{C}$. Etch rate increases with temperature, following closely the Arrhenius relationship, for temperatures above $60^{\circ} \mathrm{C}$, although with very little change in anisotropy [24]. However, since groove etching as part of a Sliver cell fabrication sequence is conducted on wafers that have undergone prior treatments such as oxidation, shallow heavy doping and surface etches and since etching is required to take place into deep, narrow grooves rather than on large area samples, it is necessary to examine real practical anisotropies for a range of groove etching scenarios by using actual etch depth and width measurements.

Table III. Measured crystal plane anisotropy of deep, narrow etched grooves using tailored TMAH solution, for different wafer pre-treatments.

\begin{tabular}{|c|c|}
\hline Wafer pre-treatment & $\begin{array}{l}\text { Anisotropy } \\
\{110\}:\{111\}\end{array}$ \\
\hline Etch 1: Oxide only & $>80$ \\
\hline Etch 1: Boron $(50 \Omega / \square)$, Oxide & $>80$ \\
\hline Etch 1: Boron $(200 \Omega / \square)$, Oxide & $>76$ \\
\hline Etch 2: Oxidation & 64 \\
\hline Etch 2: Phos, Strip Si one side, Oxide & 58 \\
\hline Etch 2: Phos, Strip Si, Boron $(50 \Omega / \square)$, Oxide & 59 \\
\hline Etch 2: Phos, Strip Si, Boron $(200 \Omega / \square)$, Oxide & 64 \\
\hline Etch 3: Oxidation & 72 \\
\hline Etch 3: Oxide, Strip Si one side, Oxide & 65 \\
\hline Etch 3: Phos, Strip Si, Boron $(50 \Omega / \square)$, Oxide & 69 \\
\hline Etch 3: Oxide, Strip, Phos, Strip, Boron, Oxide & 72 \\
\hline
\end{tabular}

The majority of high-efficiency cells fabricated at ANU have utilized a tailored TMAH solution of specific concentration and temperature to achieve best results. This solution gives a high etch rate and offers processing flexibility owing to its very high selectivity between silicon 
and common silicon dielectric masking films. Measured anisotropy is shown in Table III, where 'Phos' refers to a $\mathrm{PCOl}_{3}$ tube diffusion $(<10 \mathrm{Ohm} / \mathrm{sq})$, 'Strip Si' refers to a single-sided full area

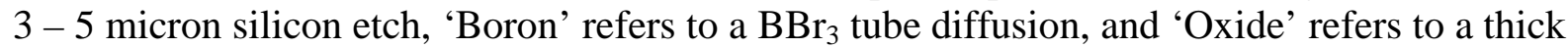
thermally grown silicon dioxide. An anisotropy of between 60 and 80 can be consistently achieved across the range of possible wafer treatments. It is worth noting that higher anisotropy values have previously been observed at ANU using KOH. Furthermore, a well-controlled etching set-up in an industrial facility using TMAH, $\mathrm{KOH}$ or $\mathrm{NaOH}$ should achieve higher anisotropy than reported here.

\section{Surface texturing}

Texturing of the sun-facing surfaces of solar cells is critical in achieving high efficiencies, through a combination of reduced reflection of incident light and improved lighttrapping. Conventional mono-crystalline cells typically use a weak anisotropic etch solution to turn planar $\{100\}$ surfaces into random pyramid textured surfaces, while for multi-crystalline cells an acid texture solution, which largely relies on the surface roughness resulting from the wire-saw wafering process, is usually employed. However for Sliver cells, which after anisotropic etch feature smooth $\{111\}$ sidewall surfaces, neither of these methods can be used. Figure 5 shows an SEM image of a $\{111\}$ wafer surface which has been textured using a new type of texturing developed at ANU by Weber and Blakers [25,26].

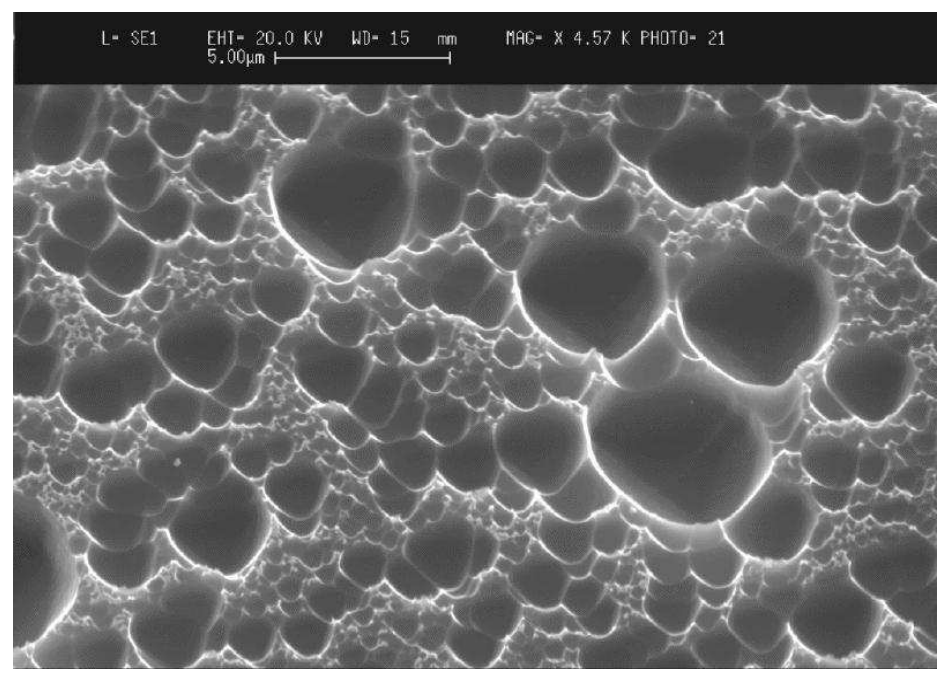

Figure 5. SEM image of textured $\{111\}$ wafer surface, using a process based on acid etching of silicon through a thin LPCVD silicon nitride.

The texturing method consists of selective random acid etching of silicon surfaces, regardless of crystal plane, utilizing the irregular nature of very thin layers of deposited nitride. A thin oxide, in the order of $20 \mathrm{~nm}$, is first grown on the surfaces to be textured. For etched sliver sidewalls this is done immediately following groove formation. A very thin (less than $10 \mathrm{~nm}$ ) silicon nitride film is then deposited onto all surfaces in an LPCVD furnace, which results in thickness non-uniformities. A 1:50 HF:HNO3 solution is then used to etch holes through the nitride, underlying oxide and then initiate texturing. The etch rate of silicon in the acid etch solution is a great deal faster than for either of the dielectrics and thus the silicon rapidly etches wherever the deposited nitride was thinnest. The acid solution is an isotropic etchant so the holes in the nitride quickly translate into rounded pits in the silicon. Spectral reflectivity measurements 
of silicon surfaces prepared using this method, with anti-reflection coating and after encapsulation are comparable to measurements for conventional texture methods.

\section{Silicon doping}

The currently preferred fabrication sequence for Sliver cells uses standard tube furnace diffusions for purposes of creating $\mathrm{p}-\mathrm{n}$ junctions and for facilitating good ohmic contacts. Owing to the unique topology of Sliver wafers two interesting challenges arise: firstly, the diffused emitter regions and the opposite polarity back surface field diffusions overlap lengthwise along the corners of the Slivers; and, secondly, the light sidewall emitter diffusion takes place over a very large surface area accessed via very narrow groove openings. The first of these issues could be avoided altogether by employing a more complex fabrication sequence which physically isolates the two doped regions. Alternatively, the possibility of formation of a tunnel-shunt can be mitigated by carefully managing each of the diffusions and subsequent high-temperature drive-in steps to ensure that one or both dopant surface concentrations are sufficiently reduced. The second challenge requires careful monitoring and control of the sidewall diffusion to ensure that an emitter is created which is relatively uniform, has good blue response and minimal recombination and has adequately low sheet resistance to ensure that resistance losses are minimized. Emitter sheet resistance can easily be the dominant loss factor for wide Sliver cells.
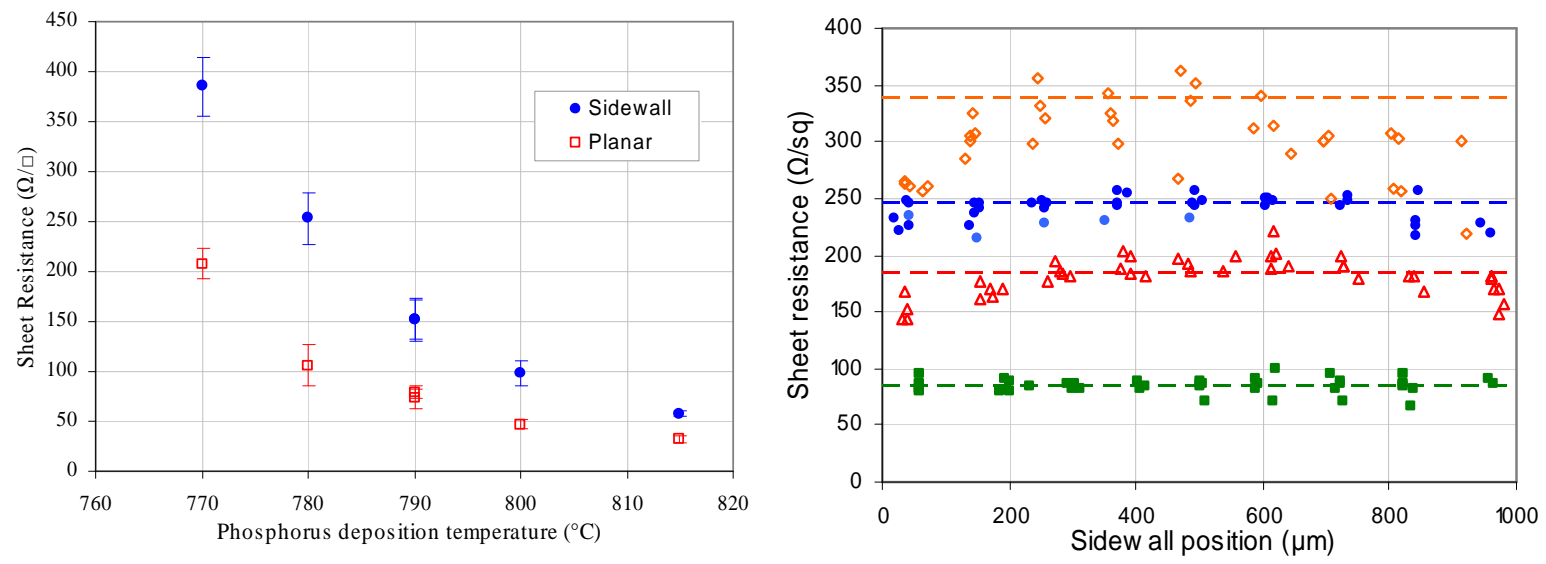

Figure 6. Sliver sidewall and planar sheet resistance measurements for a range of diffusions (left) and measured sheet resistance profile across the sidewall width (right).

During an ideal tube furnace diffusion process, the $\mathrm{POCl}_{3}$ reaction product $\left(\mathrm{P}_{2} \mathrm{O}_{5}\right)$, which supplies the source of phosphorus to the silicon, is rapidly deposited in sufficient quantity on all available surface so as to provide a virtually inexhaustible supply of dopant atoms and thus ensure uniform doping across all expose silicon surfaces. However, it proves difficult to achieve this on wafers with deep, narrow grooves. The rate of dopants diffusing into the Sliver sidewall is limited by the arrival rate of diffusion gases into and through the narrow grooves. Doping is thus considerably lighter on Sliver sidewalls than observed on normal wafer surfaces, as demonstrated in Figure 6 (left) which shows sheet resistance measurements made on planar monitor wafers and specially prepared wafers containing sidewall monitor Slivers (strips of undoped silicon equivalent to Slivers) which are diffused simultaneously. Note that a specifically calibrated four point probe method has been used for sidewall monitor Slivers once removed from the wafer after the diffusion. Sheet resistance on a sliver sidewall is typically observed to be two to three times higher than on a planar wafer, a key factor that must be taken into account when designing and monitoring diffusions for Sliver cell fabrication. Additionally, by laser- 
isolating narrow channels at different locations across these sidewall monitor Slivers, and using a similar calibrated probing method, the sheet resistance across the Sliver sidewall can be measured (Figure 6, right) and shown to be relatively uniform but slightly heavier at the Sliver edges where the diffusion gases arrive first.

\section{DISCUSSION}

Cells with high open-circuit voltage and high fill-factor have been consistently produced at ANU using the Sliver cell fabrication sequence outlined in this paper. Not only have high efficiency cells been produced but, equally importantly, the processing sequence has been shown to deliver a high yield and consistency. The highest efficiency Sliver cells that have so far been fabricated at ANU have recorded efficiencies in excess of $20 \%$ were made from $1 \mathrm{~mm}$ thick wafers, and incorporated an LPCVD SiN $(n \approx 2.1)$ anti-reflection coating. For reasons associated with ease of processing, these cells were fabricated without surface texturing but included a postprocess rear surface lambertian reflection coating applied once the cells had been removed from the wafer. A summary of characteristics for cells having undergone such treatment are presented in Table IV. Note that dozens of similar cells without the lambertian treatment were also tested, each with efficiency above $19 \%$. Calibrated in-house cell measurements only are reported. The authors believe that cell efficiencies of up to $21 \%$ are possible in an industrial setting, given the care that can be afforded even in a production environment because of the large effective cell surface area contained in each wafer.

Table IV. Measured data for six Sliver® cells fabricated at ANU.

\begin{tabular}{ccccc}
\hline $\begin{array}{c}\text { Median values for } \\
\text { tested cells }\end{array}$ & $\begin{array}{c}\mathbf{V}_{\text {oc }} \\
(\mathbf{m V})\end{array}$ & $\begin{array}{c}\mathbf{J}_{\text {sc }} \\
\left(\mathbf{m A} / \mathbf{c m}^{2}\right)\end{array}$ & FF & Eff \\
\hline @ 1 sun & 666 & 37.0 & 0.82 & $20.1 \%$ \\
Std Dev & 3.3 & 0.18 & 0.008 & $0.2 \%$ \\
\hline
\end{tabular}

An additional focus at ANU is on the use of Sliver cells in low to medium concentration ratio concentrator PV systems. Using specifically tailored processing conditions, cells have been fabricated with efficiencies of $18.8 \%$ at 9 suns (above $18.6 \%$ between 5 and 15 suns) and $18.4 \%$ at 37 suns (above 18.2\% between 30 and 50 suns) respectively. These cells had no texturing and oxide only anti-reflection coatings. Incorporation of sidewall texturing and $\mathrm{SiN}$ anti-reflection coatings would yield efficiencies exceeding $20 \%$ for similar cells. The highest recorded efficiency for a $103.5 \mathrm{~cm} 2$ Sliver cell module is $17.7 \%$.

The processing sequence described in this paper is one of many possible fabrication sequences that could be used. Transform Solar, currently undertaking a major commercialization effort with the Sliver technology, is using a quite different processing sequence. Development of a sequence using novel processing techniques with the potential for greatly reduced processing time and cost in production is now underway. The goal is to reduce processing complexity and wet chemistry steps while preserving or improving solar cell efficiency.

Finally, for a commercially successful Sliver cell fabrication facility a streamlined, high throughput high-yield cell fabrication sequence is only one half of the challenge. It is also essential that a fast, reliable and inexpensive Sliver module assembly process also be developed. Given the unique size of Sliver cells, a solar module constructed from Sliver cells will contain in the order of ten thousand, compared with 70 to 80 conventional solar cells. Furthermore, owing to the physical properties of Sliver cells, many aspects of cell-handling and module assembly cannot be used. The many techniques and methods developed specifically for the construction 
and assembly of Sliver modules are not the subject of this paper. Needless to say, highly refined, complex and robust processes are required to extract, evaluate, lay-up, interconnect and encapsulate many hundreds to thousands of individual Sliver cells to form a module. An example of a Sliver module and also a proto-type flexible module, which exploits the flexible properties of very thin silicon, are shown in Figure 7.
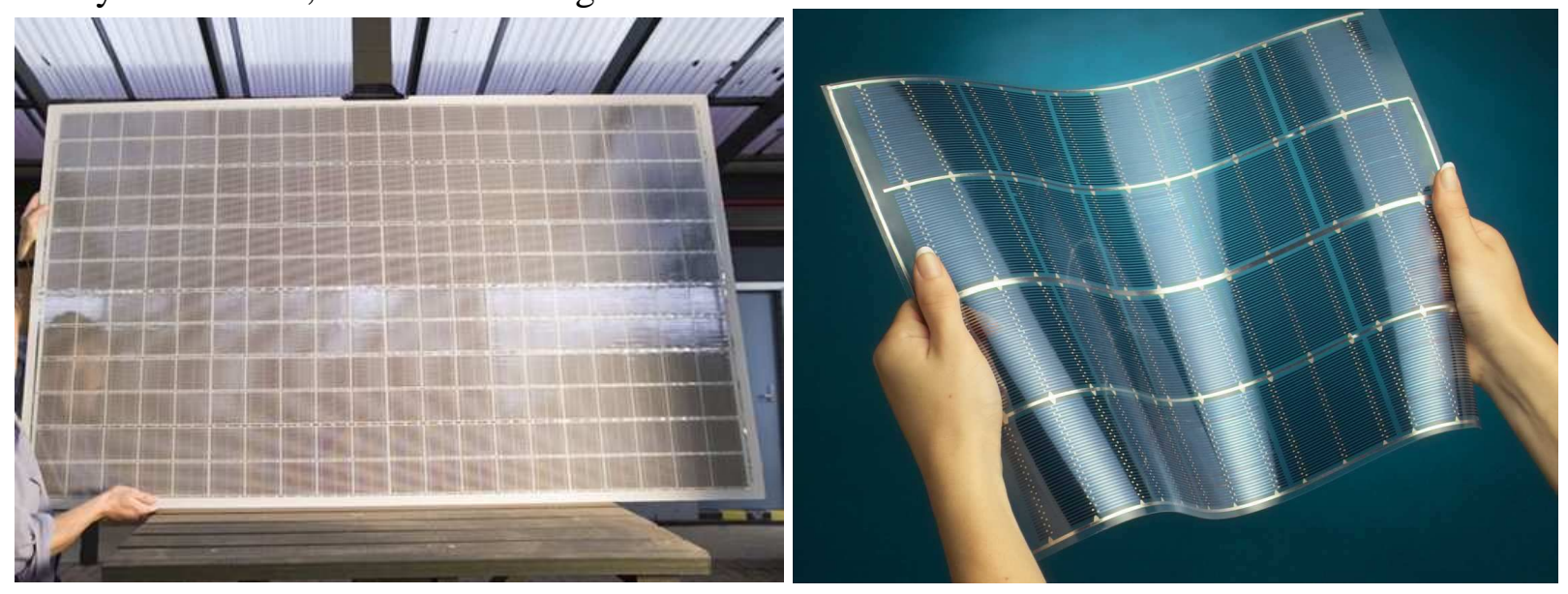

Figure 7. A Sliver module (left) and a flexible proto-type module (right).

\section{CONCLUSIONS}

Sliver technology is a novel high-efficiency solar cell technology which takes advantage of the many properties of crystalline silicon solar cells while offering some of the benefits of thin-film technology in terms of vastly reduced material usage. Many hundreds to thousands of Sliver solar cells can be manufactured in a single conventional mono-crystalline wafer, via a critical micromachining groove formation step and a series of tailored but standard semiconductor processes. Sliver cells have been fabricated with efficiencies exceeding $20 \%$, with module efficiencies of greater than $17 \%$ anticipated. Current Sliver technology research at ANU is directed towards simplifying the fabrication process and improving cell efficiencies through the use of state-of-the-art photovoltaic processing techniques. A robust, high-yield industrial Sliver cell fabrication sequence and module production process offers the potential for low cost photovoltaic products. Currently, the company Transform Solar is undertaking a major commercialization effort with Sliver technology, having transferred manufacturing to a former semiconductor facility in the US. Products will be launched late in 2011.

\section{ACKNOWLEDGMENTS}

The authors would like to acknowledge the support of the project commercial partners, Transform Solar. Transform Solar is currently undertaking commercial development of Sliver technology, and the current Sliver technology research at ANU is being conducted in partnership with the company. This project has been supported by the Australian Government through the Australian Solar Institute (ASI), part of the Clean Energy Initiative. The Australian Government, through the ASI, is supporting Australian research and development in solar photovoltaic and solar thermal technologies to help solar power become cost competitive with other energy sources. The views expressed herein are not necessarily the views of the Australian Government, and the Australian Government does not accept responsibility for any information or advice contained herein. 


\section{REFERENCES}

1. G. Hering, 'Cell Production Survey 2010, Photon International Magazine, 03/2011 p.186-218, (2011)

2. Solarbuzz, 'Marketbuzz 2011', accessed at www.solarbuzz.com [last accessed 24 March 2011], (2011)

3. C. Breyer, C. Birkner, et al., 'Research and development investments in PV - a limiting factor for a fast PV diffusion?', Proceedings of the $25^{\text {th }}$ PV solar Energy Conference, Valencia, Spain, (2010)

4. T. Roder, S. Eisele, et al., 'Add-on laser tailored selective emitter solar cells', Progress in Photovoltaics, vol. 18: p.505-510, (2010)

5. H. Antoniadis, F. Jiang, W. Shan and Y. Liu, 'All screen-printed mass produced silicon ink selective emitter solar cells', Proceedings of the $35^{\text {th }}$ IEEE PV Specialists Conference', Honolulu, USA, (2010)

6. D. Kray, N. Bay et al., 'High-efficiency large area inducstrial LCP selective emitter solar cells ready for production', Proceedings of the $25^{\text {th }}$ PV solar Energy Conference, Valencia, Spain, (2010)

7. Z. Shi, S. Wenham and J. Ji, 'Mass production of the innovative PLOTO solar cell technology', Proceedings of the $34^{\text {th }}$ IEEE PV Specialists Conference', Philadelphia, USA, (2009)

8. E. Schmich, Y. Gassenbauer, et al., 'Industrial multi-crystalline silicon solar cells with dielectrically passivated rear side and efficiencies above 18\%', Proceedings of the $25^{\text {th }} \mathrm{PV}$ solar Energy Conference, Valencia, Spain, (2010)

9. E. Kerschaver and G. Beaucarne, 'Back-contact solar cells: a review', Progress in Photovoltaics, vol. 14: p.107-123, (2006)

10. MWT or similar cell ref, 'Screen-printed emitter-wrap-through solar cell with single step side selective emitter with 18.8\% efficiency', Progress in Photovoltaics, Article in print / published online August 2010, (2010)

11. M. Green, 'The Path to 25\% Silicon Solar Cell Efficiency: History of Silicon Cell Evolution', Progress in Photovoltaics, vol. 17: p.183-189, (2009)

12. M. Green, 'Estimates of Te and In Prices from Direct Mining of Known Ores', Progress in Photovoltaics, vol. 17: p.347-359, (2009)

13. S. Reber, A. Eyer, et al., 'Progress in Crystalline Silicon Thin-Film Solar Cell Work at Fraunhofer ISE', 20th European Photovoltaics Solar Energy Conference, Barcelona, (2005)

14. B. Terheiden, R. Horbelt, and R. Brendel, 'Thin-film Solar Cells and Modules from the Porous Silicon Process Using 6" Si Substrates', 21st European Photovoltaic Solar Energy Conference, Dresden (2006)

15. A. Aberle, 'Progress in Evaporated Crystalline Silicon Thin-Film Solar Cells on Glass', 4th World Conference on Photovoltaic Energy Conversion, Hawaii, USA, (2006)

16. K.J. Weber and A.W, Blakers, Semiconductor Processing PCT/AU01/01546, 2001

17. K.J. Weber, A.W. Blakers, M.J. Stocks, J. H. Babaei, V.A. Everett, A.J Neuendorf, and P.J. Verlinden, “A Novel Low Cost, High Efficiency Micromachined Silicon Solar Cell”, Electron Device Letters 25, 37, 2004

18. A.W. Blakers, M.J. Stocks, K.J. Weber, V. Everett, J. Babaei, P. Verlinden, M. Kerr, M. Stuckings and P. Mackey, "Sliver® Solar Cells", 13th NREL workshop on Crystalline Si Materials and Processing, Vail Colorado, 2003

19. K.J. Weber, A.W. Blakers, V. Everett and E. Franklin, "Results of a cost model for Sliver® cells", 21st EC Photovoltaic Solar Energy Conference, Dresden, September 2006

20. Transform Solar website, www.transformsolar.com, [last accessed 24 March 2011], (2011)

21. K.J. Weber, J. MacDonald, V.A. Everett, P.N.K. Deenapanray, M.J. Stocks and A.W. Blakers, "Modelling of Sliver® Modules incorporating a lambertian rear reflector", 19th European Photovoltaic Solar Energy Conference, Paris, 2004

22. E. Franklin, V. Everett, A. Blakers \& K. Weber, 'Sliver Solar Cells: high-efficiency, low-cost PV technology', Advances in OptoElectronics, Volume 2007, Article ID 35383, doi:10.1155/2007/35383, (2007)

23. M. Shikida, K. Sato, K. Tokoro, and D. Uchikawa, 'Differences in anisotropic etching properties of KOH and TMAH solutions', Sensors and actuators, vol. 80: p.179-188, (2000).

24. E. Steinsland, T. Finstad, and A. Hanneborg, 'Etch rates of (100), (111) and (110) single-crystal silicon in TMAH measured in-situ by laser reflectance interferometry', Sensors and actuators, vol. 86: p.73-80, (2000)

25. K. Weber and A. Blakers, Patent number: US2005104163, 'Semiconductor Texturing Process', US Patent Office, [Country: USA], (2005).

26. K. Weber and A. Blakers, 'A Novel Silicon Texturisation Method Based on Etching Through a Silicon Nitride Mask', Progress in Photovoltaics, vol. 13: p.691-695, (2005). 